\title{
On-Chip Bondwire Magnetics with Ferrite-Epoxy Glob Coating for Power Systems on Chip
}

\author{
Jian Lu, Hongwei Jia, Andres Arias, Xun Gong, and Z. John Shen \\ School of Electrical Engineering and Computer Science, University of Central Florida, Orlando, FL 32816, USA \\ Correspondence should be addressed to Z. John Shen, johnshen@mail.ucf.edu
}

Received 3 January 2008; Accepted 13 May 2008

Recommended by Ty McNutt

A novel concept of on-chip bondwire inductors and transformers with ferrite epoxy glob coating is proposed to offer a cost effective approach realizing power systems on chip (SOC). We have investigated the concept both experimentally and with finite element modeling. A $Q$ factor of 30-40 is experimentally demonstrated for the bondwire inductors which represents an improvement by a factor of 3-30 over the state-of-the-art MEMS micromachined inductors. Transformer parameters including self- and mutual inductance and coupling factors are extracted from both modeled and measured $S$-parameters. More importantly, the bondwire magnetic components can be easily integrated into SOC manufacturing processes with minimal changes and open enormous possibilities for realizing cost-effective, high-current, high-efficiency power SOCs.

Copyright (C) 2008 Jian Lu et al. This is an open access article distributed under the Creative Commons Attribution License, which permits unrestricted use, distribution, and reproduction in any medium, provided the original work is properly cited.

\section{INTRODUCTION}

System on Chip (SOC) is an emerging trend of integrating all components of an electronic system including digital, analog, mixed-signal, communication, and sensor functions, into a single integrated circuit. The SOC concept embodies what many believe to be the ultimate level of integration: an entire system on one chip. Since its emergence in the 1990s, the SOC concept has gained wide acceptance in a broad range of applications from supercomputing to embedded systems. The proliferation of the SOC concept into power management systems has also generated a great deal of interest in the electronics industry. Power management is a key enabling technology behind the digital revolution. Each year hundreds of millions of power converters are manufactured and embedded into computers, telecommunication equipment, consumer products, automobiles, and industrial control systems worldwide. Unfortunately, the power management auxiliary subsystem sometimes takes as much as $50 \%$ of the board space of the main electronic system. For this reason, power management SOCs that monolithically integrate all active and passive components using low-cost semiconductor manufacturing processes will provide an extremely attractive solution with significant improvement in performance and unprecedented reduction in board space, parts count, and time-to-market. The power SOC concept is particularly well received in several fast growing power management markets such as point of load (POL) dc/dc converters, LED drivers, and battery-powered mobile applications.

Unfortunately, the development of power management SOC's is seriously hindered by a few major technical barriers including integration of integrating magnetic passive components. The main challenge is to find a cost effective means of integrating inductors and transformers onto the silicon chip, while achieving adequate performance in terms of inductance, $\mathrm{dc}$ series resistance, maximum saturation current, coupling coefficient, and $Q$ factor. Current research work on integrated magnetics for power SOC's has predominantly focused on utilizing microelectromechanical system (MEMS) micromachining technology as a postprocessing step after the completion of the CMOS chip containing all power switching devices and control circuitry [1-8]. Sophisticated MEMS technology allows sequential deposition and patterning of numerous layers of conductor, insulator, permalloy, or ferrite thin films to form desirable inductor and transformer structures. However, the high dc resistance (typically 0.5 to $5 \Omega$ ) and poor $Q$ factor (typically 3 to 8 ) of the MEMS inductors/transformers severely limit the current handling capability and efficiency of the power SOC. 
More critically, the large increase of fabrication complexity and cost associated with the MEMS postprocessing approach raises questions on its feasibility to facilitate large-scale commercialization of the power SOC concept into the extremely cost-sensitive power supply market.

In this paper, we propose and investigate a new costeffective approach to form on-chip magnetic components utilizing existing bondwires with additional ferrite epoxy glob coating formed during the packaging process.

\section{BASIC CONCEPT}

Wire bonding is commonly used in nearly all power management IC packages today. As shown in Figure 1, thin aluminum or gold bondwires of 25 to $250 \mu \mathrm{m}$ in diameter are used to provide necessary electrical interconnection between the silicon chip and the package leads. It is well known that the bondwires of IC packages typically exhibit a parasitic inductance of a few $\mathrm{nH}$ and a resistance of several to several tens of $m \Omega$. On-chip bondwire inductors were first explored in RF integrated circuits by Craninckx and Steyaert in 1995 [9]. Although bondwires act as natural inductors, the inductance and coupling effect are typically insufficient for power converter applications. A higher inductance in the range of several tens of $\mathrm{nH}$ is required for power SOC (in conjunction with increased switching frequencies to a range of $10-30 \mathrm{MHz}$ ). Several US patents have addressed this issue by suggesting the use of multiple bondwires connected in series [10-13] but suffer from the drawback of increased chip area.

We proposed to add ferrite epoxy composite glob coating to the bondwires to increase inductance and improve coupling effect as shown in Figure 2. Bondwire inductors and transformers can be made between the chip and package leads or between pads on the chip [14-16]. Unlike traditional ferrite ceramics, ferrite epoxy materials are essentially ceramic magnetic powders mixed with a polymer binder and can be dried or cured at temperatures less than $200^{\circ} \mathrm{C}$. These materials combine appropriate magnetic properties with a high resistivity and high manufacturability $[17,18]$. The ferrite epoxy glob core can be formed to cover the bondwires during the SOC packaging process by brushing, squeegeeing, dipping, dripping, inking, or other viable dispensing techniques using high precision robotic tools similar to the commonly used electronic assembly equipment such as solder paste dispensers.

Compared to the prior art MEMS inductor technology, the proposed approach has the following advantages.

(i) All SOC components (i.e., control circuitry, power switches, gate drivers, feedback compensation networks, etc.) except for the magnetics are fabricated with standard silicon processing technology, eliminating the need for costly post-CMOS MEMS processing steps.

(ii) The on-chip bondwire inductors and transformers can be integrated into the power SOC packaging process with minimal changes. This opens enormous

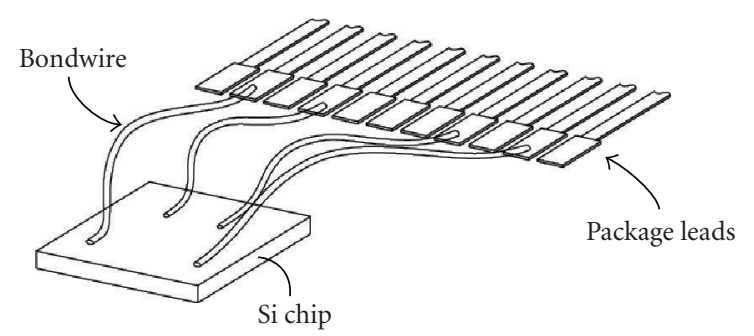

FIGURE 1: A packaged IC chip interconnected to metal leads through bondwires.

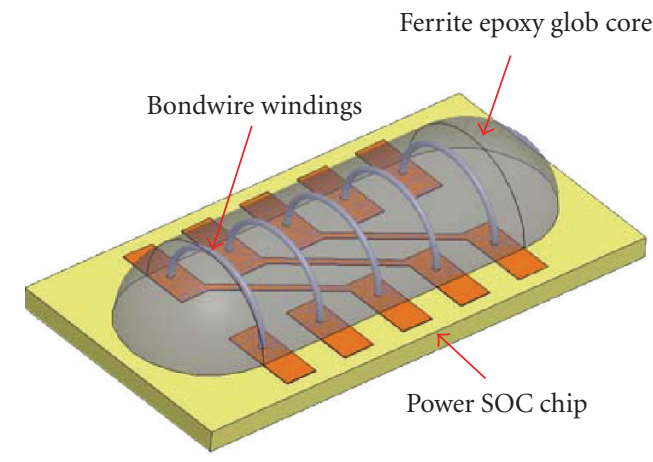

FIGURE 2: Concept of on-chip bondwire magnetic component with ferrite epoxy glob core.

possibilities for realizing cost-effective, high current, high efficiency power SOC's.

(iii) Aluminum or gold bondwires, due to their relatively large diameters, are much more conductive than the thin metal films in MEMS inductors. Therefore, a much lower dc resistance and higher $Q$ factor can be expected for the bondwire inductors. Quality factor $Q$ is the ratio of reactive impedance to equivalent series resistance (or ESR), an important parameter of inductor/transformer performance. High $Q$ leads to low-power dissipation and high efficiency of the power converter.

(iv) The electromagnetic field of a bondwire inductor or transformer is mainly distributed outside the silicon substrate. The Eddy current loss in the silicon substrate at high frequency, a major concern in MEMS magnetics, can therefore be minimized.

\section{FINITE ELEMENT MODELING}

The proposed bondwire inductor and transformer structures were modeled and analyzed using the electromagnetic simulation tool HFSS from Ansoft, Pa, USA [19]. HFSS is a highperformance full-wave electromagnetic (EM) finite element simulator for arbitrary $3 \mathrm{D}$ passive component modeling. The objective here is to investigate the effect of ferrite epoxy glob coating on the inductance value and coupling effect of bondwires using EM simulation. Furthermore, EM modeling helps optimizing bondwire inductor and transformer design 
in terms of physical dimensions of bondwire, ferrite coating shape, coating position, coating thickness, and the ferrite material type used.

\subsection{Inductors}

HFSS simulation generates a set of $S$ parameters from the inductor structure which are then converted to a set of $Y$ parameters. The effective quality factor $Q$ and inductance $L$ can be then extracted from the $Y$ parameters by using the following equations:

$$
\begin{aligned}
L_{\text {eff }} & =-\frac{1}{\omega \operatorname{Im}\left(y_{11}\right)}, \\
Q & =-\frac{\operatorname{Im}\left(y_{11}\right)}{\operatorname{Re}\left(y_{11}\right)} .
\end{aligned}
$$

Figure 3 shows the modeled inductance value of bondwire inductors with a ferrite epoxy coating thickness of 0 (bare wire), 10, 20, 30, and 40 mils over a frequency range from 1 to $500 \mathrm{MHz}$. The bondwire is $17.5 \mathrm{~mm}$ long and $20 \mathrm{mil}(0.51 \mathrm{~mm})$ in diameter. A permeability of 16 is assumed for the ferrite epoxy material in the simulation work. The inductance value of the bondwire increases from $14 \mathrm{nH}$ without ferrite coating to over $70 \mathrm{nH}$ with a ferrite coating of $40 \mathrm{mil}$ in thickness. A five times increase in inductance value is therefore observed.

A set of measurement data is included in Figure 3 for comparison with the modeling result. A reasonably good agreement can be seen between the modeling and measurement results. Key bondwire inductor design parameters include length and diameter of the bondwire, and thickness and permeability of the ferrite epoxy coating. It is observed in Figure 3 that the inductance of the bondwire inductor increases with ferrite epoxy coating thickness since a larger ferrite thickness leads to a smaller magnetic reluctance, greater magnetic flux, and greater inductance. However, there are practical limitations on how much ferrite epoxy coating can be applied into the IC package which has a limited space.

Figure 4 shows the modeled inductance value of bondwire inductors with a ferrite epoxy permeability of 1 (bare wire), 9, 16, 25, 36, and 49 over a frequency range from 1 to $500 \mathrm{MHz}$. Naturally, the inductance value increases with increasing permeability of the ferrite epoxy material. Iron oxide-based ferrite ceramic materials usually demonstrate a relative permeability up to a few thousands. Ferrite epoxy materials with mixed ferrite powders and organic binder tend to have a much lower effective permeability in a range of 9 to 20 depending on the loading percentage of the ferrite powders. For example, the permeability of the ferrite epoxy used in our experimental study from Methode Development Company (Chicago, Ill, USA) is between 12 and 16. However, it is possible that new high- $\mu$ ferrite epoxy materials can be developed in the future. The simulation results indicate that the inductance of a bare bondwire can be increased by a factor of 10 if a $\mu$ value of 50 can be used.

The effect of bondwire diameter on its inductance value is also studied. Figure 5 shows the HFSS simulated trend of

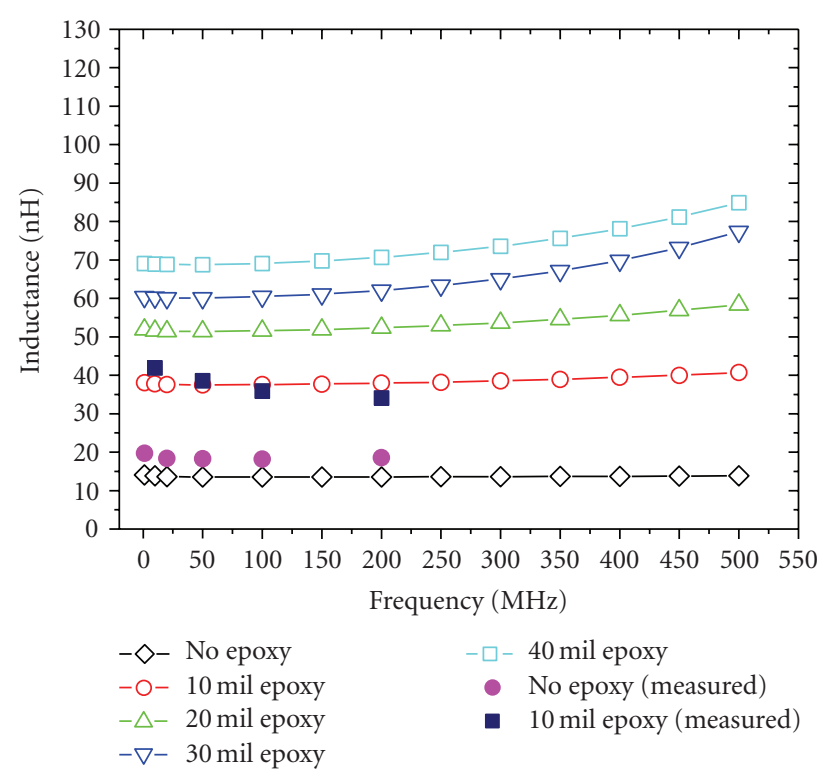

FIGURE 3: Effect of ferrite epoxy coating thickness on the inductance value of a bondwire based on HFSS simulation. Measurement data is also included for comparison. The bondwire is $17.5 \mathrm{~mm}$ long and 20 mil in diameter. A $\mu$ value of 16 is assumed.

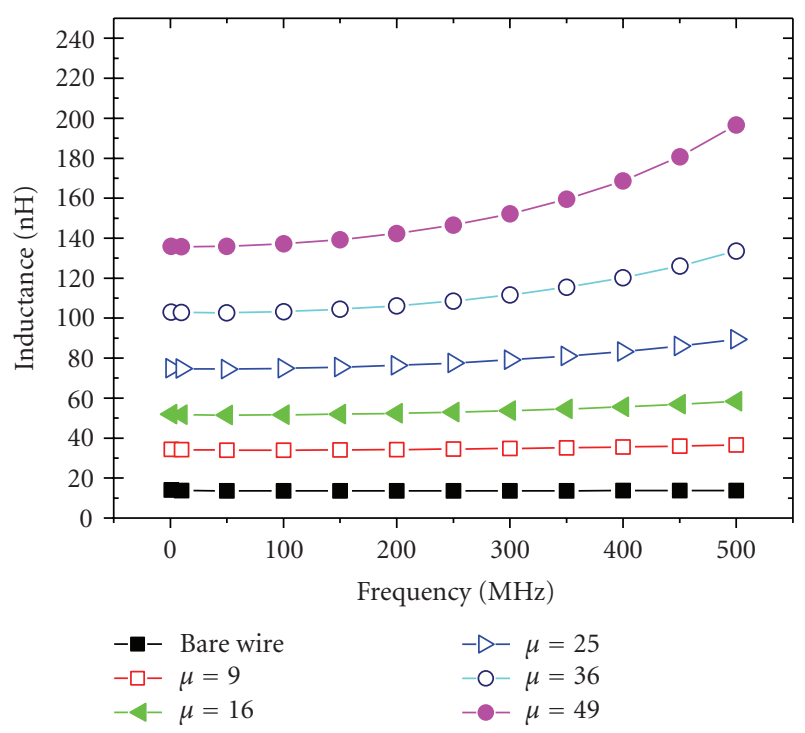

FIGURE 4: Effect of ferrite epoxy coating permeability on the inductance value of a bondwire based on HFSS simulation. The bondwire is $17.5 \mathrm{~mm}$ long and $20 \mathrm{mil}$ in diameter. A thickness of 20 mils is assumed for the ferrite epoxy coating.

inductance increase with decreasing wire diameter. However, it is observed that the inductance increase is not significant. Furthermore, a small diameter of the bondwire leads to a high dc series resistance and a small $Q$ factor. Therefore, it may not be an effective solution to increase the inductance by using very thin bondwires in power SOC's. 


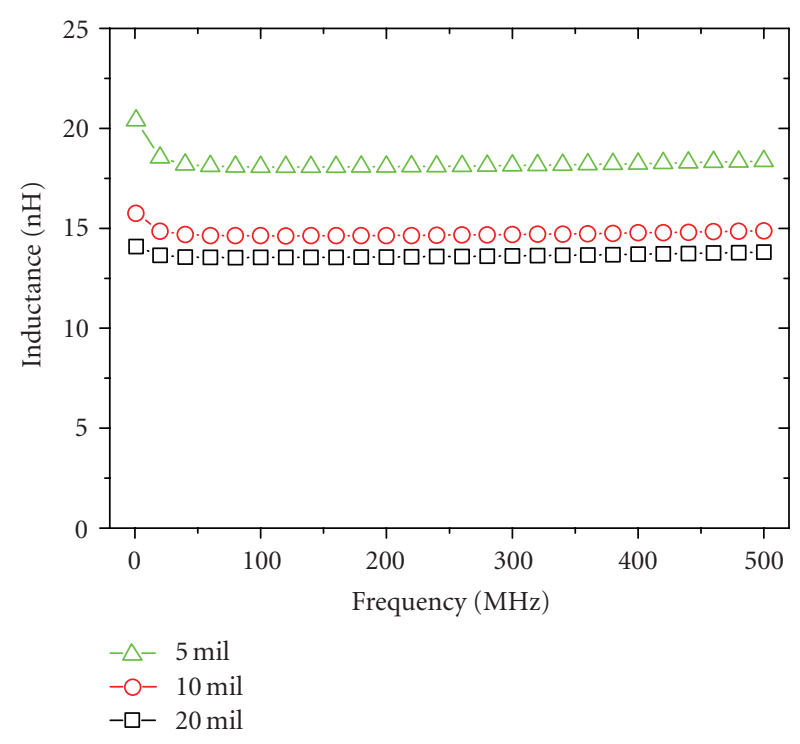

FIGURE 5: Inductance of bondwires with a diameter of 5, 10, and 20 mils based on HFSS simulation. The bondwire is $17.5 \mathrm{~mm}$ long. There is no ferrite epoxy coating.

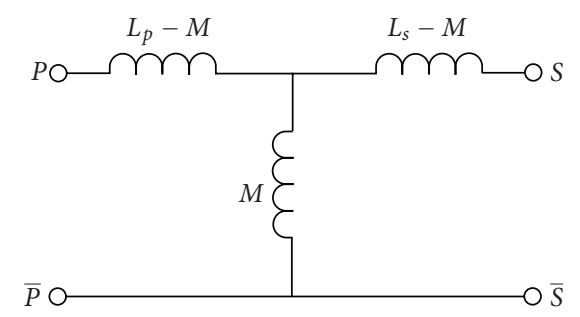

Figure 6: A two-port transformer T-model.

In short, the EM simulation work indicates that the bondwire inductance can be increased by increasing the thickness and permeability of the ferrite epoxy coating, and to a less extent, by using thinner bondwires.

\subsection{Transformers}

Self inductance, mutual coupling coefficient, series dc resistance, and $Q$ factor are among the most important properties of a power transformer. In general, a high mutual coupling coefficient and a high self inductance along with low dc series resistance are desirable. We first study those parameters with a modeling and analysis approach. Figure 6 shows a two-port transformer model that we use in our study for analysis and parameter extraction. $L_{p}, L_{s}$, and $M$ are the primary selfinductance, secondary self-inductance, and mutual inductance, respectively. The $T$-section model in Figure 6 uses three inductors to model the mutual coupling between the primary and secondary windings. This model considerably simplifies analysis of circuits containing transformers [20].
The turn ratio $T$, mutual inductance $M$, primary inductance $L_{p}$, and secondary inductance $L_{s}$ are given by

$$
\begin{aligned}
T & =\sqrt{\frac{L_{p}}{L_{s}}}, \\
L_{p(\text { leakage })_{-}} & =L_{p}-M=L_{p} \cdot(1-k), \\
L_{s(\text { leakage })} & =L_{s}-\frac{M}{T^{2}}=L_{s} \cdot(1-k), \\
M & =k \sqrt{L_{p} L_{s}}=k L_{p}, \quad \text { if } L_{p}=L_{s},
\end{aligned}
$$

where $k$ is the coupling coefficient $(0 \leq k \leq 1)$ and can be extracted from modeling and measurement data.

Transformer parameters can be simply extracted from the two-port network $Z$-parameters as the following:

$$
\begin{aligned}
L_{p} & =\frac{\operatorname{Im}\left(Z_{11}\right)}{\omega}, \\
L_{s} & =\frac{\operatorname{Im}\left(Z_{22}\right)}{\omega}, \\
Q_{p} & =\frac{\operatorname{Im}\left(Z_{11}\right)}{\operatorname{Re}\left(Z_{11}\right)}, \\
Q_{s} & =\frac{\operatorname{Im}\left(Z_{22}\right)}{\operatorname{Re}\left(Z_{22}\right)}, \\
k & =\sqrt{\frac{\operatorname{Im}\left(Z_{12}\right) \cdot \operatorname{Im}\left(Z_{21}\right)}{\operatorname{Im}\left(Z_{11}\right) \cdot \operatorname{Im}\left(Z_{22}\right)} .}
\end{aligned}
$$

The $Z$-parameters of the transformer network can be converted from its $S$-parameters from electromagnetic field simulation or network analyzer measurement. The $S$ parameters from HFSS simulation of the two-port transformer can be described as the following.

$|S 11|^{2}$ is the reflected power from Port 1.

$|S 12|^{2}$ is the transmitted power from Port 1 to Port 2.

$|S 21|^{2}$ is the transmitted power from Port 2 to Port 1.

$|S 22|^{2}$ is the reflected power from Port 2.

For two-port transformers, the transmission coefficient is simply $S 21$ which indicates the capability of the transformer to transfer power from the primary side to the secondary side.

It is found from our modeling analysis that the spacing between the bondwires has a great influence to the transformer parameters. A small spacing improves the transformer's performance significantly. Figure 4 illustrates the simulated self-inductance, mutual inductance, and quality factor of $1: 1$ bondwire transformer with spacing of $15 \mathrm{mil}$ between the two bondwires. A maximum $Q$ factor of 138 is observed.

Figures 8 and 9 show the coupling coefficient $k$ and power transmission parameter $S 21$ extracted from HFSS simulation for a bondwire spacing ranging from 12 to 50 mils. It is observed that both parameters improve as the distance between the bondwires decreases. This is indeed encouraging since the advanced wire bonding machines used in today's microelectronics manufacturing can deliver 


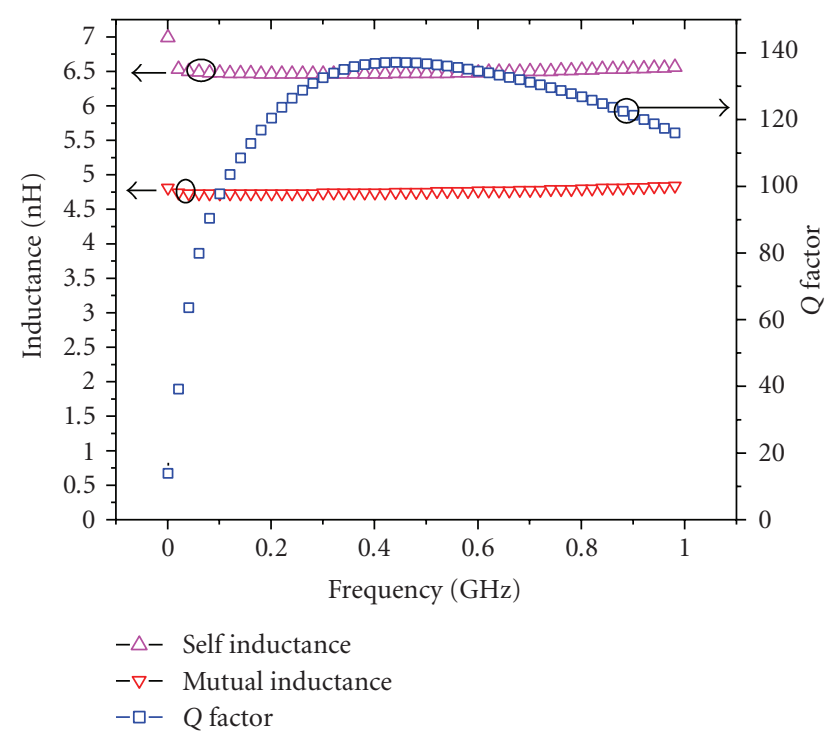

FIgURE 7: Simulated self inductance, mutual inductance, and quality factor of a 15 mil spacing bondwire transformer.

a bondwire spacing as small as 1 mil, which can guarantee excellent coupling between bondwires.

\section{EXPERIMENTAL}

We have conducted experimental investigation of the proposed on-chip bondwire magnetics concept using two types of ferrite epoxy composite materials. The first material is a custom formulated magnetic epoxy comprised of manganese-zinc $(\mathrm{MgZn})$ ferrite powder with an average particle size of $10 \mu \mathrm{m}$, thermaoplastic resin, and solvent from Methode Development Corporation. The manganese-zinc ferrite loading powder is commercially available (Steward $73300)$. The average surface area of the powder is $1.4 \mathrm{~m} 2 / \mathrm{g}$. The saturation moment of the bulk powder is $79.4 \mathrm{emu} / \mathrm{g}$. The cured ferrite composite (no solvent) consisted of $96 \%$ by mass ferrite with the balance consisting of polymer. The effective permeability is between 12 and 16 . The second material is a ferrite nanocomposite from Inframat Corporation, Conn, USA, that is comprised of very fine $(\mathrm{NiZn}) \mathrm{Fe}_{2} \mathrm{O}_{4}$ nanoparticles with an average size of $5-15 \mathrm{~nm}$ and a commercial epoxy.

The test reported herein was conducted on standalone copper and aluminum wires initially and then on aluminum bondwires bonded onto a PCB substrate. Copper wires with a fixed length of $20 \mathrm{~mm}$ but two different diameters of $250 \mu \mathrm{m}(10 \mathrm{mil})$ and $500 \mu \mathrm{m}(20 \mathrm{mil})$ were used to emulate the bondwires in IC packages. Note that copper has a slightly lower resistivity than more commonly used gold or aluminum. The ferrite epoxy materials were manually brushed onto the copper bondwire to form a ferrite bead. Curing consisted of a thermal treatment of the ferrite beads in an oven at $140^{\circ} \mathrm{C}$ for 30 minutes for both ferrite epoxy material options. The ferrite-polymer composites display negligible conductivity and therefore are electrically self-

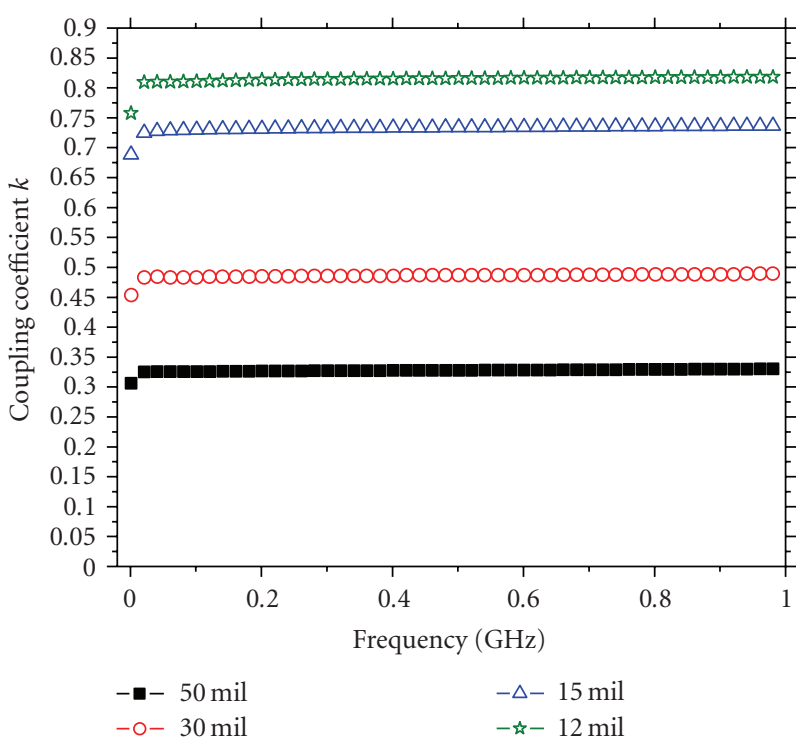

FIGURE 8: Coupling coefficient $k$ for variable bonding spacing.

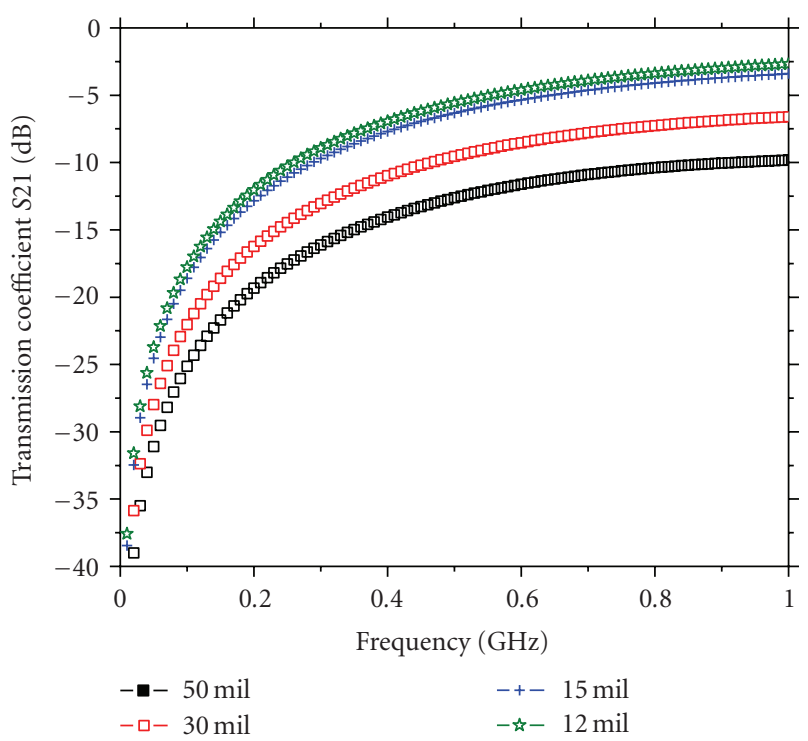

Figure 9: Transmission coefficient $S 21$ for various bondwire spacing.

isolated from the bare copper bondwires. Figure 10 shows a photo of aluminum bondwire transformer with and without the ferrite epoxy coating on a PCB substrate. The wire bonding was conducted on an Orthodyne M20 wirebonder using 5 mil aluminum bondwires.

\subsection{Inductors}

The bondwire inductors were characterized with an HP 4284. A high precision LCR meters in a low frequency range up to $1 \mathrm{MHz}$. DC resistance was measured with an Instek $801 \mathrm{H}$ milli-Ohm meter. High frequency measurement was performed using an Agilent 8753 S-parameter network 


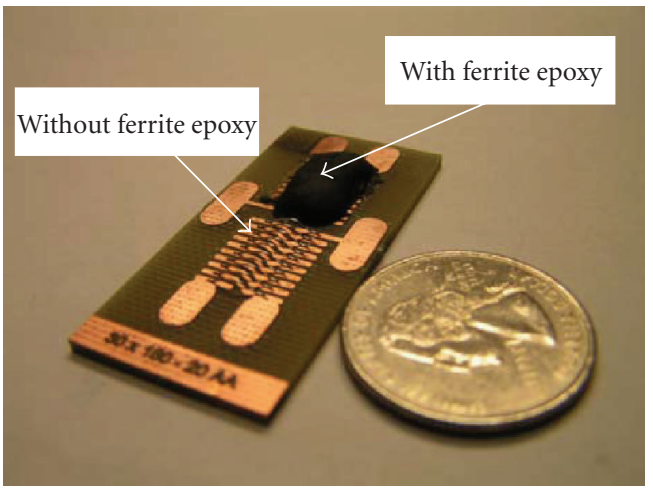

Figure 10: Fabricated bondwire transformer $\left(N_{p}: N_{s}=9: 1\right)$.

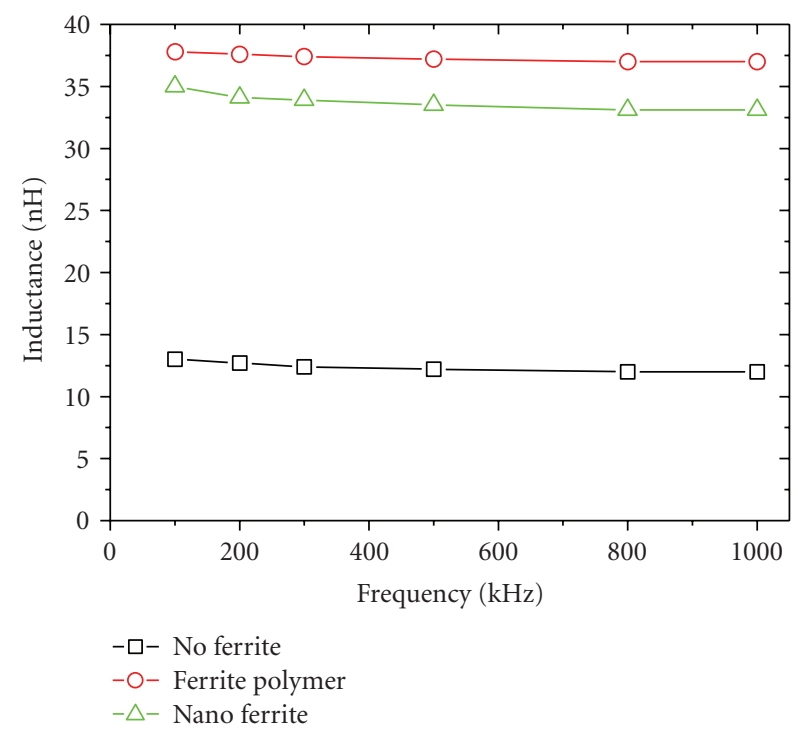

FIGURE 11: Measured inductance versus frequency for a bare copper wire and wires coated with two different ferrite epoxy materials. The wire is $20 \mathrm{~mm}$ long and $10 \mathrm{mil}$ in diameter.

analyzer. The effective quality factor $Q$ and inductance $L$ can be then extracted from the $Y$ parameters by using (1).

Figures 11 and 12 show the measured inductance for the $10 \mathrm{mil}$ and $20 \mathrm{mil}$ bondwire inductors with no ferrite epoxy coating, the ferrite polymer bead, and the ferrite nanocomposite bead, respectively. The inductance of bare copper wires was increased by a factor of 2.8 to 3.5 with the addition of the ferrite epoxy coating. The dc resistance of the $10 \mathrm{mil}$ and $20 \mathrm{mil}$ bondwire inductors was measured as $7.1 \mathrm{~m} \Omega$ and $1.7 \mathrm{~m} \Omega$ respectively using an Instek $801 \mathrm{H}$ milliOhm meter. The 10 mil bondwire inductor demonstrates an inductance of $38 \mathrm{nH}$ and a dc resistance of $7.1 \mathrm{~m} \Omega$. Figure 13 compares the $Q$-factor of the state-of-the-art MEMS microinductors, commercial wire-wound inductors, and the bondwire inductors in this work. The bondwire inductors demonstrate a $Q$ factor of 30-40 in a frequency range of 2 to $20 \mathrm{MHz}$, similar to that of commercial wirewound inductors, but significantly higher than that of the MEMS microinductors. Figure 14 compares the state-

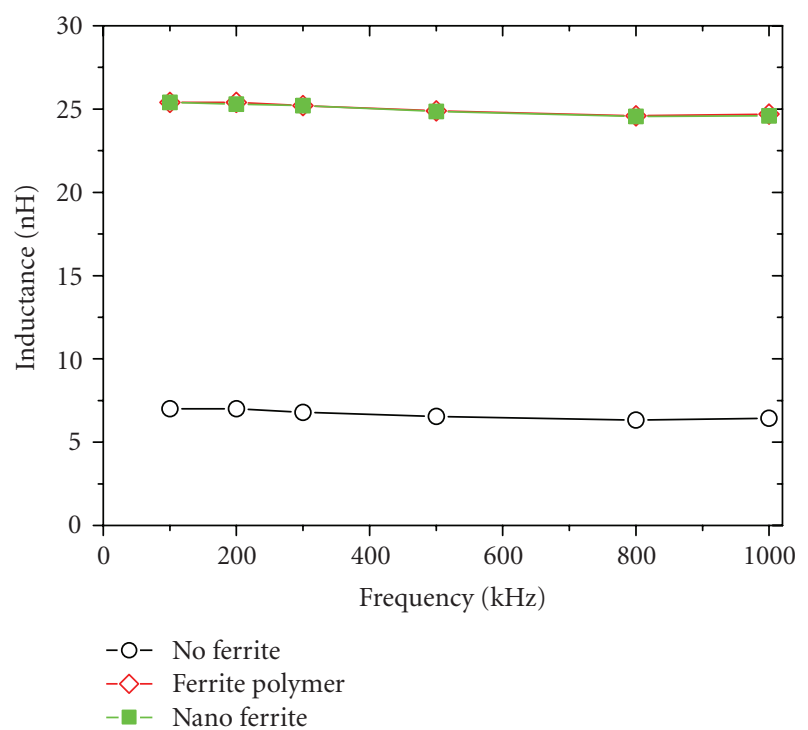

FIGURE 12: Measured inductance versus frequency for a bare copper wire and wires coated with two different ferrite epoxy materials. The wire is $20 \mathrm{~mm}$ long and $20 \mathrm{mil}$ in diameter.

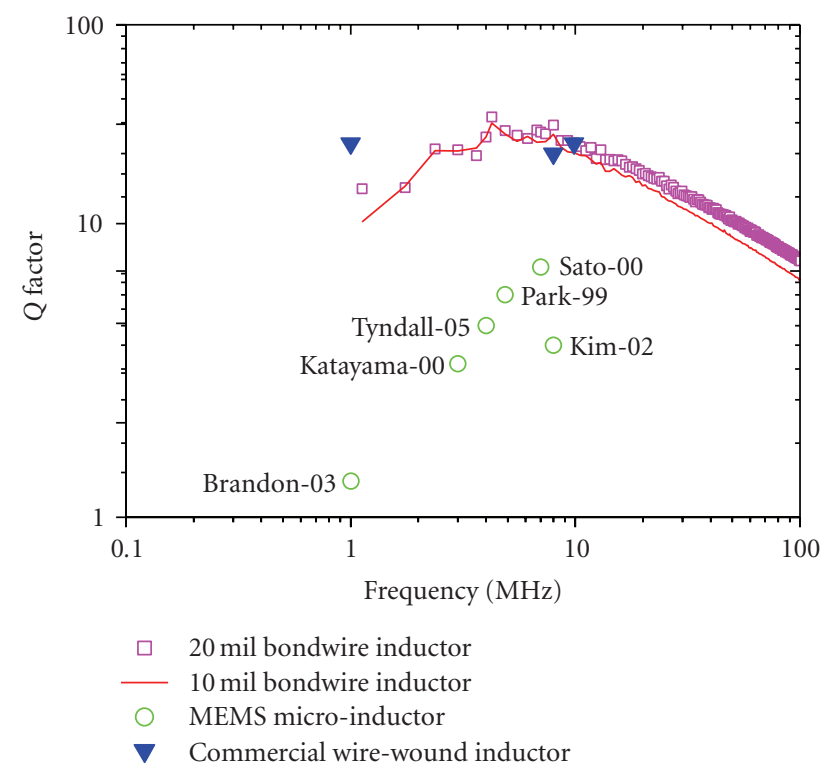

FIgURE 13: Comparison of $Q$ factors between the state-of-the-art MEMS micro-inductors, commercial wire wound inductors, and the bondwire inductors.

of-the-art MEMS microinductors, commercial wire-wound inductors, and the bondwire inductors in this work in terms of inductance and dc winding resistance achieved. It is clearly shown that the bondwire inductors offer a solution for high current power SOC applications in which the MEMS microinductors fall short. Figure 15 shows the measured inductance of the bondwire inductors over a high frequency range, indicating a self-resonant frequency between 700 and $800 \mathrm{MHz}$. The bondwire inductors were also tested in a pulsed switching circuit for core saturation characterization. Figure 16 shows measured switching 


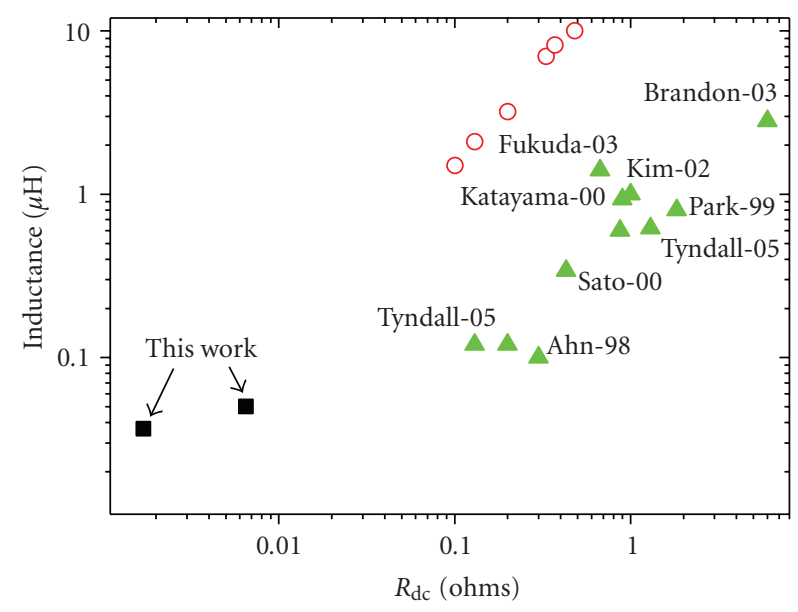

- Bondwire inductor

○ Commercial wire-wound inductor

$\triangle$ MEMS micro-inductor

FIgURE 14: Comparison of the state-of-the-art MEMS microinductors, commercial wire wound inductors, and the bondwire inductors in terms of inductance and dc resistance.

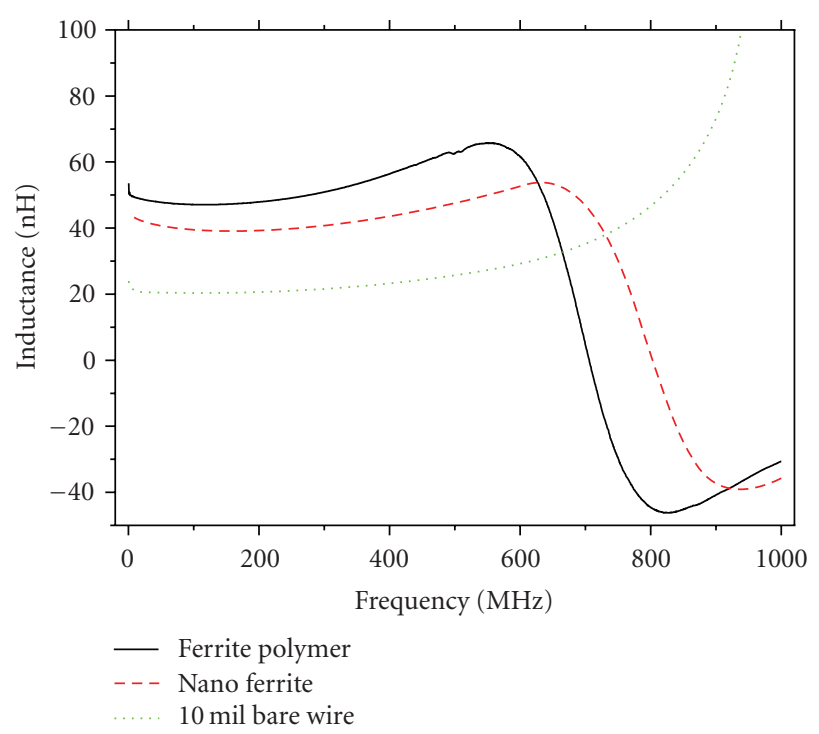

FIgURE 15: Measured high-frequency inductance for the $10 \mathrm{mil}$ bondwire inductors with and without ferrite beads.

waveforms of the inductor current. No noticeable core saturation phenomenon was observed for an inductor load current up to 47A, most likely due to the distributed gaps between the ferrite particles in the ferrite epoxy materials.

\subsection{Transformers}

We have conducted experimental investigation of the proposed on-chip bondwire transformer concept with and without the ferrite epoxy composite materials. Figures 17 and 18 show the primary and secondary voltage waveforms of a 9 : 1 bondwire transformer without and with ferrite epoxy core at $16 \mathrm{MHz}$. It is observed that the bondwire transformer

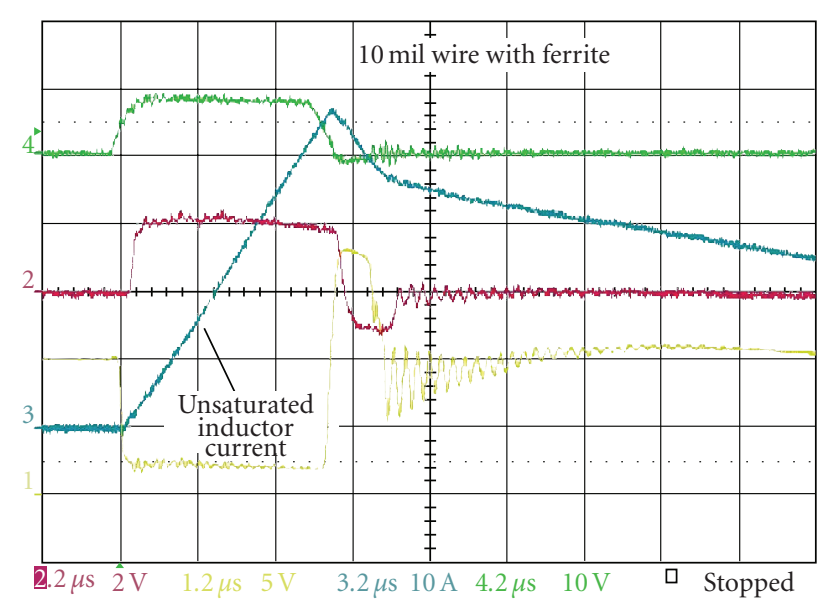

FIGURE 16: Measured switching waveform of the 10 mil bondwire inductors for core saturation characterization.

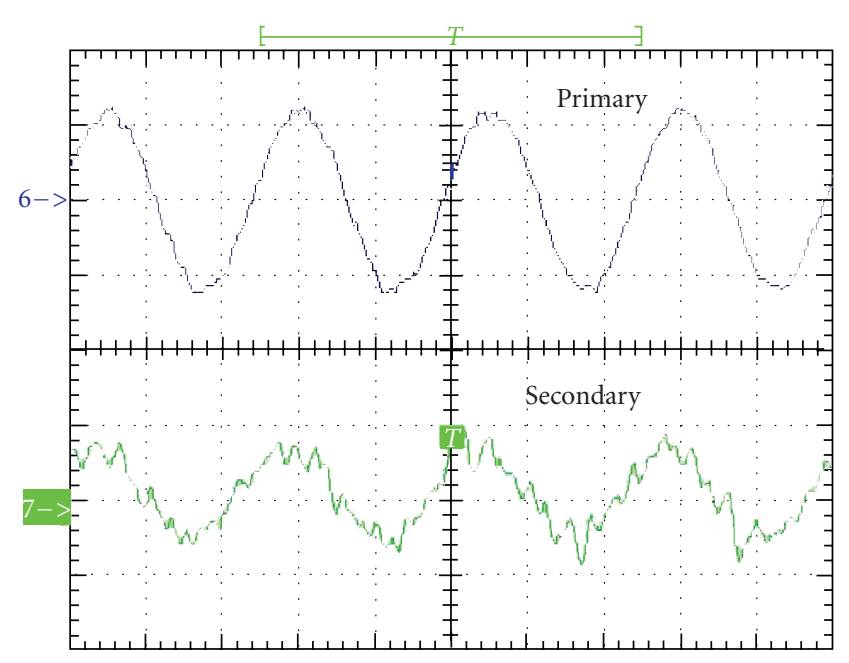

6) [TPS2024].CH1 $20 \mathrm{mV} 25 \mathrm{~ns}$
7) [TPS2024].CH3 $5 \mathrm{mV} 25 \mathrm{~ns}$

FIGURE 17: Measured primary and secondary voltage waveforms without ferrite epoxy core $(9: 1)$.

with ferrite epoxy core demonstrated less distortion in the secondary voltage waveform than its counterpart without ferrite core.

It is very difficult to characterize transformers in time domain at high frequencies due to the parasitic effects. We use a $S$-parameter network analyzer to extract all transformer parameters such self- and mutual inductances, $k$-factor, and S21 parameter. The PCB substrate was designed as $50 \Omega$ transmission lines to minimize the input reflection. A Cascade Microtech's (Ore, USA) M150 probe station is used to test the bondwire transformers on PCB substrates. Two FPC-1000 probes are used for two-port measurement. The Fixed-Pitch Compliant (FPC)-Series fixtures are high frequency $50 \Omega$ coaxial probe that offers a signal line with two low-inductance fixed-pitch ground contacts (GSG). The PCB designs are based on detailed HFSS simulation, and work 


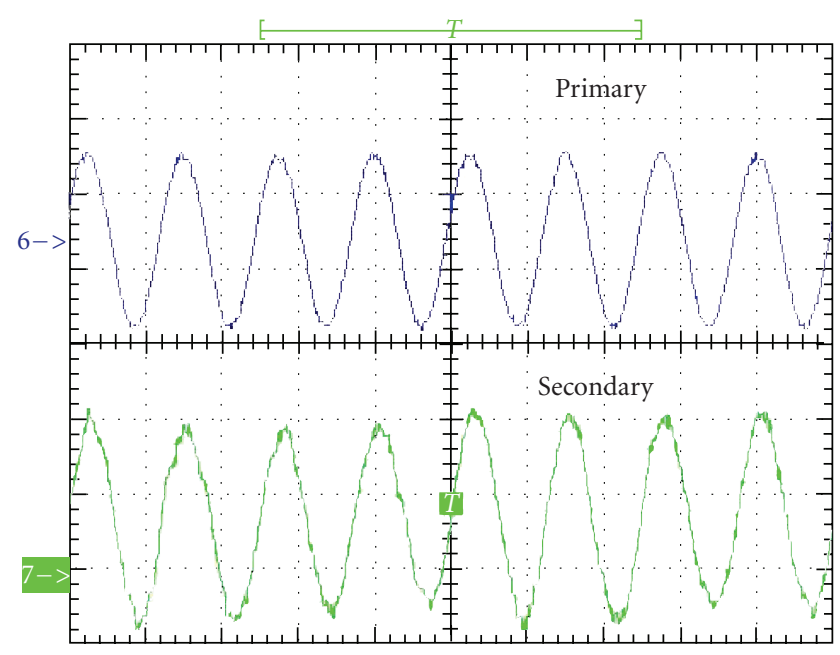

6) [TPS2024].CH1 $100 \mathrm{mV} 50 \mathrm{~ns}$

7) [TPS2024].CH3 $2 \mathrm{mV} 50 \mathrm{~ns}$

FIGURE 18: Measured primary and secondary voltage waveforms with ferrite epoxy core $(9: 1)$.

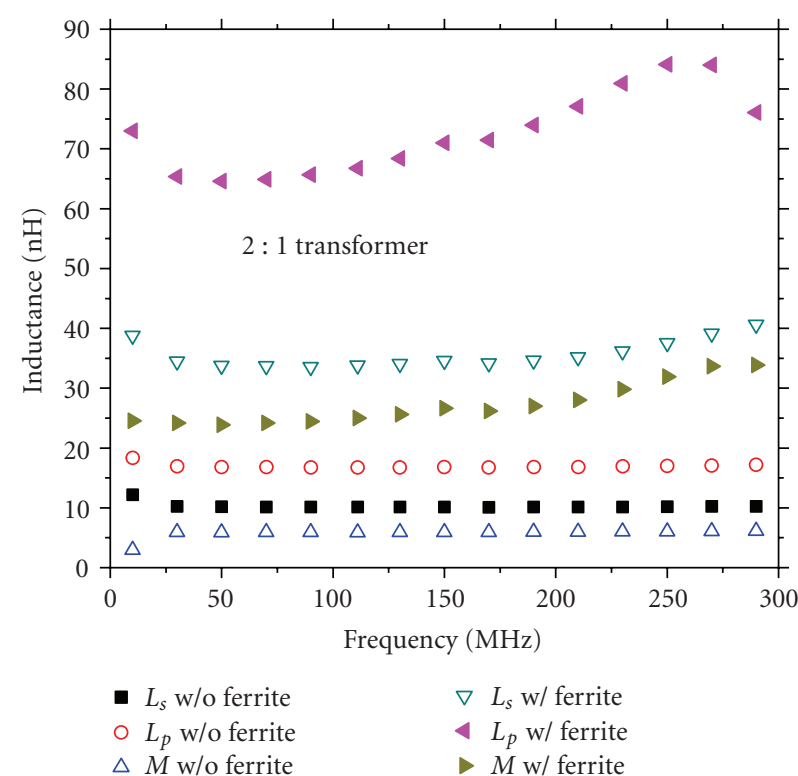

FIGURE 19: Self inductance and mutual inductance measurement data for bondwire 2:1 transformers with and without ferrite core.

well with the FPC-1000 probes. A PNA E8361A Network Analyzer receives input from the two FPC-1000 probes and is used to measure the $S$-parameters of the transformers.

Figure 19 shows the extracted primary self-inductance $L_{p}$, secondary self-inductance $L_{s}$ and mutual inductance $L$ with and without the ferrite epoxy core for a $2: 1$ transformers. The dc resistance of each bondwire is around $7 \mathrm{~m} \Omega$. It is observed that all inductances increase after ferrite epoxy glob is applied.

Figure 20 shows the extracted $k$-factor with and without the ferrite epoxy core for the 2 : 1 transformers. It is observed that the $k$-factor increased from 0.46 to 0.6

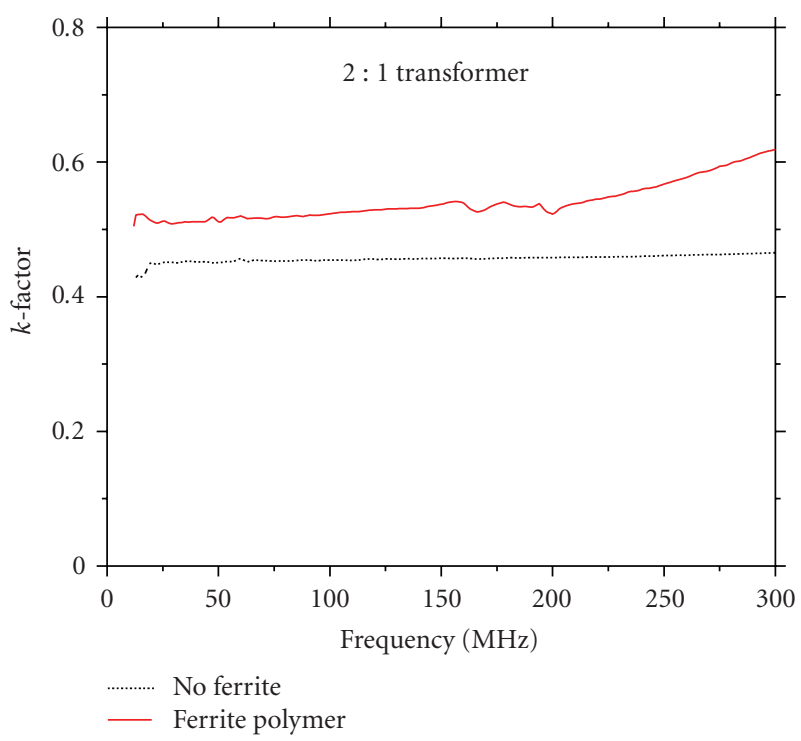

FIgURE 20: Measured $k$-factor of a $2: 1$ bondwire transformer before and after applying ferrite epoxy.

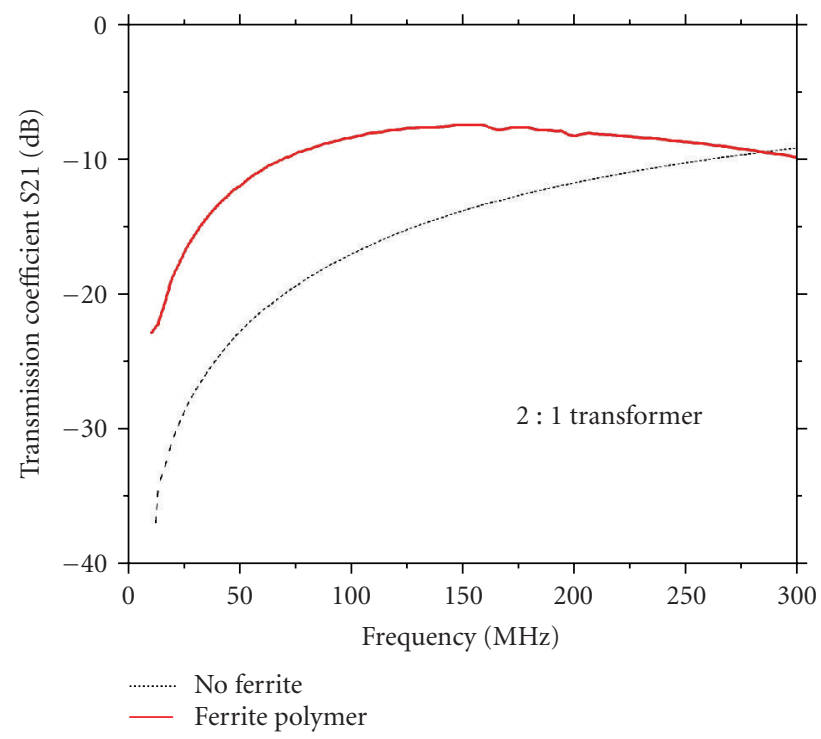

FIGURE 21: Measured transmission coefficient S21 of a 2 : 1 bondwire transformer before and after applying ferrite epoxy.

(30\% improvement) for $2: 1$ transformer. This shows the effectiveness of ferrite epoxy core. Figure 21 illustrates the transmission coefficient $S 21$ of the $2: 1$ transformer. $S 21$ is a critical parameter which determines the transformer's power transferring capability. In very high frequency span, since the coupling coefficient $k$ is no longer meaningful, $S 21$ is the only performance parameter for the transformer.

The most important finding from this experiment is that the coupling effect of the bondwire transformers can be significantly improved with the application of ferrite epoxy core glob. Not only can we increase the $k$-factor, but also can we boost the transmission coefficient $S 21$. We experimentally 
demonstrated that the performance of the on-chip bondwire transformer can be improved by ferrite polymer epoxy globe in a frequency range of $10 \mathrm{MHz}$ to $300 \mathrm{MHz}$, which is of interest for future-generation, high frequency, high density switching power converters.

\section{CONCLUSIONS}

We have proposed and investigated the feasibility of a new concept of realizing on-chip inductors and transformers in power SOC's using existing bondwires with additional ferrite epoxy glob coating. We have investigated the concept both experimentally and with finite element modeling. A $Q$ factor of 30-40 is experimentally demonstrated for the bondwire inductors which represents an improvement by a factor of 3-30 over the state-of-the-art MEMS micromachined inductors. Transformer parameters including self- and mutual inductance, and coupling factors are extracted from both modeled and measured $S$-parameters. It is demonstrated that the performance of the bondwire transformers can be improved using ferrite polymer epoxy globe. More importantly, the bondwire magnetic components can be easily integrated into SOC manufacturing processes with minimal changes, and open enormous possibilities for realizing costeffective, high current, high efficiency power SOC's. Future work includes studies on the selection of ferrite materials, the influence of the shape and volume of the ferrite beads, the effect of the height and length of the bondwire loop, and possible implementation methods in mass production.

\section{ACKNOWLEDGMENTS}

This work was supported in part by the U.S National Science Foundation under Award no. ECS-0454835 and a grant from Intel Corporation, Calif, USA. The authors wish to thank Mr. Emil Millas and Mr. Frank St. John from Methode Development Company and Dr. Danny Xiao from Inframat Corporation, Conn, USA, for providing ferrite epoxy materials and valuable input.

\section{REFERENCES}

[1] S. C. O. Mathuna, T. O'Donnell, N. Wang, and K. Rinne, "Magnetics on silicon: an enabling technology for power supply on chip," IEEE Transactions on Power Electronics, vol. 20, no. 3, pp. 585-592, 2005.

[2] T. Sato, K. Yamasawa, H. Tomita, T. Inoue, and T. Mizoguchi, "Planar power inductor using FeCoBN magnetic film with high saturation magnetization and high electrical resistivity," in Proceedings of the 4th International Power Electronics Conference (IPEC '00), pp. 303-308, Tokyo, Japan, April 2000.

[3] C. H. Ahn and M. G. Allen, "Micromachined planar inductors on silicon wafers for MEMS applications," IEEE Transactions on Industrial Electronics, vol. 45, no. 6, pp. 866-876, 1998.

[4] Y. Fukuda, T. Inoue, T. Mizoguchi, S. Yatabe, and Y. Tachi, "Planar inductor with ferrite layers for DC-DC converter," IEEE Transactions on Magnetics, vol. 39, no. 4, pp. 2057-2061, 2003.

[5] E. J. Brandon, E. Wesseling, V. White, C. Ramsey, L. Del Castillo, and U. Lieneweg, "Fabrication and characterization of microinductors for distributed power converters," IEEE Transactions on Magnetics, vol. 39, no. 4, pp. 2049-2056, 2003.

[6] J. Y. Park, S. H. Han, and M. G. Allen, "Batch-fabricated microinductors with electroplated magnetically anisotropie and laminated alloy cores," IEEE Transactions on Magnetics, vol. 35, no. 5, part 3, pp. 4291-4300, 1999.

[7] Y. Katayama, S. Sugahara, H. Nakazawa, and M. Edo, "Highpower-density MHz-switching monolithic DC-DC converter with thin-film inductor," in Proceedings of the 31st Annual IEEE Power Electronics Specialists Conference (PESC '00), vol. 3, pp. 1485-1490, Galway, Ireland, June 2000.

[8] K. H. Kim, J. Kim, H. J. Kim, S. H. Han, and H. J. Kim, "A megahertz switching DC/DC converter using FeBN thin film inductor," IEEE Transactions on Magnetics, vol. 38, no. 5, pp. 3162-3164, 2002.

[9] J. Craninckx and M. S. J. Steyaert, "A 1.8-GHz CMOS lowphase-noise voltage-controlled oscillator with prescaler," IEEE Journal of Solid-State Circuits, vol. 30, no. 12, pp. 1474-1482, 1995.

[10] K. S. Yeo, H. P. Ian, J. Ma, M. A. Do, and J. K. W. Chew, "High performance RF inductors and transformers using bonding technique," US patent 6998953, Feburary 2006.

[11] D.-B. Cheon, "Inductor including bonding wires," US patent 6194774, Feburary 2001.

[12] R. B. Merrill and I. Singh, "Bonding wire inductor for use in an integrated circuit package and method," US patent 5886393, March 1999.

[13] M. A. Imam and S. Yoganathan, "Inductor formed at least partially in a substrate," US patent 5767563, June 1998.

[14] Z. J. Shen, "A Method of Manufacturing Low Cost onchip magnetic components," patent pending, US provisional patent, August 2006.

[15] Z. J. Shen, J. Lu, X. Cheng, H. Jia, and X. Gong, "On-chip bondwire inductor with ferrite-epoxy coating: a cost-effective approach to realize power systems on chip," in Proceedings of the 38th Annual IEEE Power Electronics Specialists Conference (PESC '07), pp. 1599-1604, Orlando, Fla, USA, June 2007.

[16] J. Lu, H. Jia, A. Arias, X. Gong, and Z. J. Shen, "Onchip bondwire transformers for power SOC applications," in Proceedings of the 23rd Annual IEEE on Applied Power Electronics Conference and Exposition (APEC'08), pp. 199-204, Austin, Tex, USA, February 2008.

[17] E. J. Brandon, E. E. Wesseling, V. Chang, and W. B. Kuhn, "Printed microinductors on flexible substrates for power applications," IEEE Transactions on Components and Packaging Technologies, vol. 26, no. 3, pp. 517-523, 2003.

[18] P. M. Raj, P. Muthana, T. D. Xiao, et al., "Magnetic nanocomposites for organic compatible miniaturized antennas and inductors," in Proceedings of the 10th International Symposium on Advanced Packaging Materials: Processes, Properties and Interfaces, pp. 272-275, Irvine, Calif, USA, March 2005.

[19] Ansoft Corporation, http://www.ansoft.com.

[20] J. R. Long, "Monolithic transformers for silicon RF IC design," IEEE Journal of Solid-State Circuits, vol. 35, no. 9, pp. 13681382, 2000. 

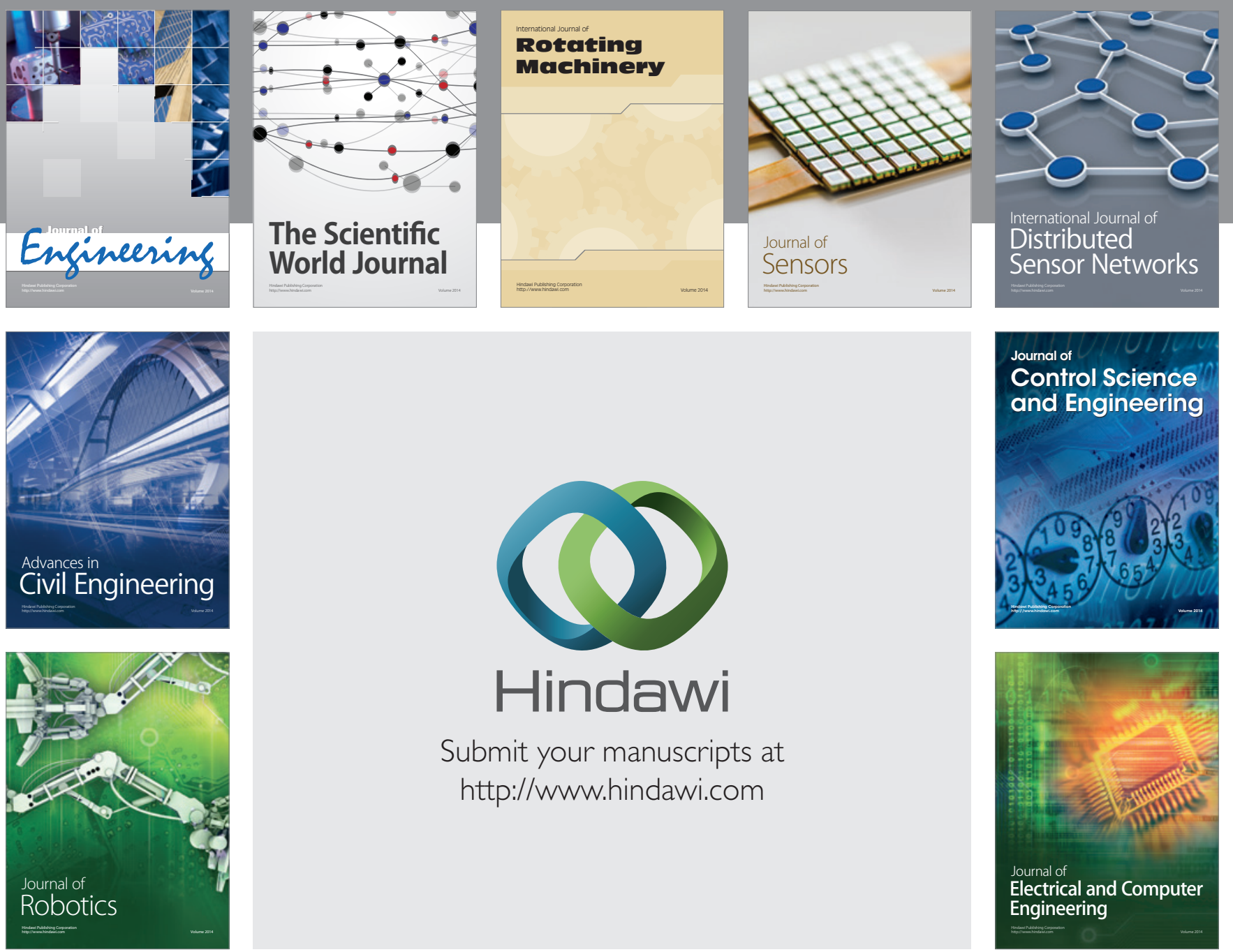

Submit your manuscripts at

http://www.hindawi.com
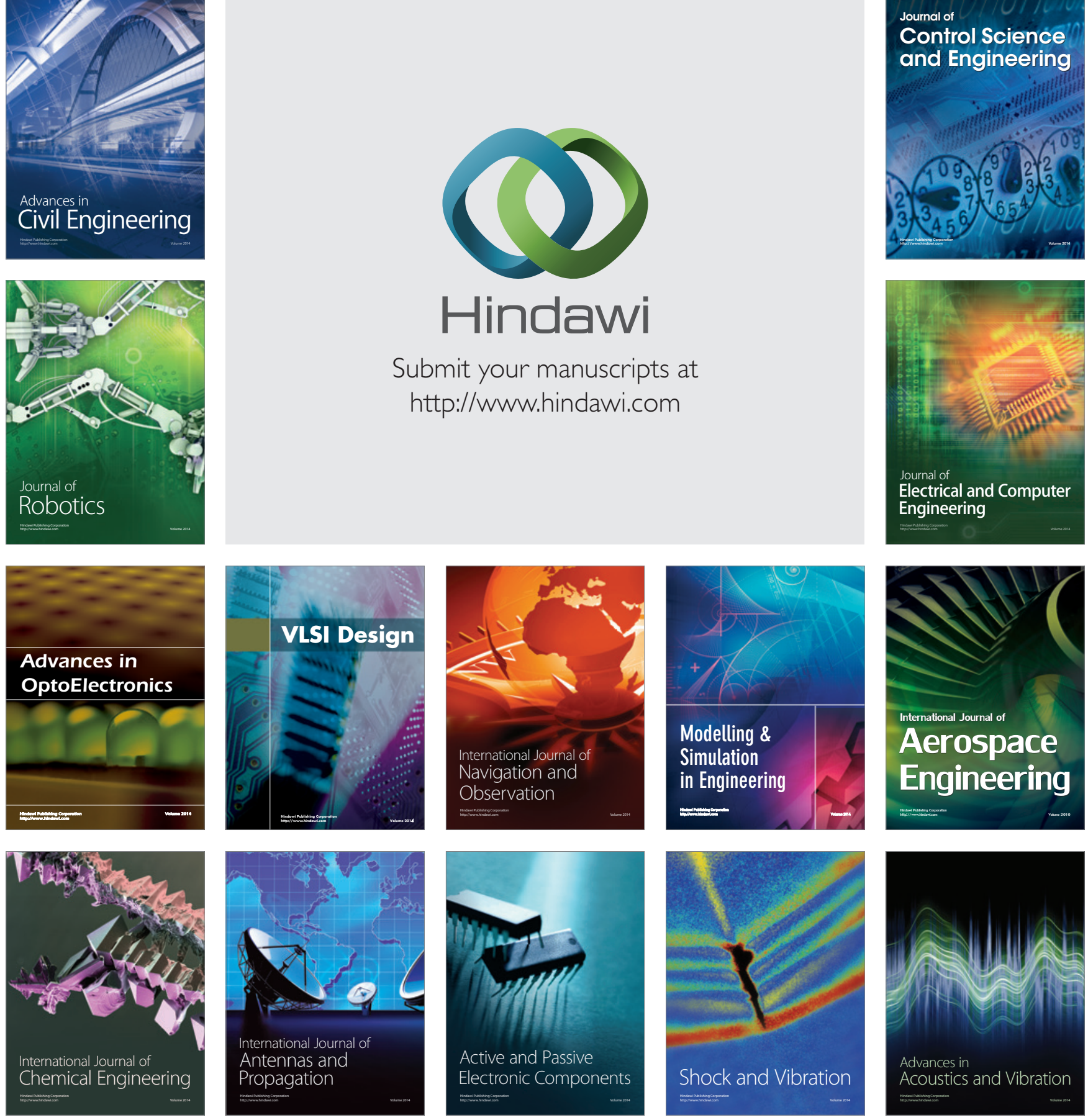